Cahiers de recherches médiévales

Journal of medieval studies

$18 \mid 2009$

Le système d'enseignement occidental $\left(\mathrm{Xl}^{\mathrm{e}}-\mathrm{XV|}{ }^{\mathrm{e}}\right.$ siècle)

\title{
Faire carrière dans une université de province
}

Caen aux $\mathrm{XV}^{\mathrm{e}}$ et $\mathrm{XVI}^{\mathrm{e}}$ siècles

Lyse Roy

\section{(2) OpenEdition}

Journals

Édition électronique

URL : https://journals.openedition.org/crm/11690

DOI : $10.4000 / \mathrm{crm} .11690$

ISSN : 1955-2424

Éditeur

Honoré Champion

Édition imprimée

Date de publication : 20 novembre 2009

Pagination : 133-144

ISSN : 1272-9752

Référence électronique

Lyse Roy, «Faire carrière dans une université de province », Cahiers de recherches médiévales [En ligne], 18 | 2009, mis en ligne le 15 décembre 2012, consulté le 15 décembre 2022. URL : http://

journals.openedition.org/crm/11690 ; DOI : https://doi.org/10.4000/crm.11690 


\title{
酷RM
}

\section{Faire carrière dans une université de province : Caen aux $X V^{\mathrm{e}}$ et $X V I^{\mathrm{e}}$ siècles}

\begin{abstract}
This article analyses the process through which professors became 'professionals' in the University of Caen during the $15^{\text {th }}$ and $16^{\text {th }}$ centuries. The process was closely linked to the financing of the University and therefore to its relations with publics powers. The Parlement of Rouen imposed two reforms (1521 and 1586) on the University in an attempt to regulate the professors more closely; these led to a definition of the professors' competence and to the assertion of the necessity of a salary for their work. This last question was central and provoked important changes in the relations between professors at the end of the $16^{\text {th }}$ century, when the University was trying to recover from the Religious Wars.

Résumé : Le processus de professionnalisation des régents de l'université de Caen aux $X V^{e}$ et $X V I^{e}$ siècles est étroitement lié au financement de l'Université et, par conséquent, à ses relations avec les pouvoirs publics. L'université de Caen se voit en effet imposer deux réformes par le parlement de Rouen, en 1521 et en 1586. Ces réformes visent notamment un meilleur encadrement des professeurs et exigent d'eux plus de discipline. Ce faisant, les critères de compétence des professeurs se définissent, et la nécessité pour eux de recevoir une rétribution régulière s'affirme. La question du salaire des professeurs provoque ainsi d'importants changements dans les relations entre les professeurs à la fin du XVI siècle, alors que l'Université tente de se relever du marasme dans lequel l'ont plongée les guerres de Religion.
\end{abstract}

Agents essentiels de la transmission du savoir, les professeurs assurent la vocation intellectuelle de l'université. Si plusieurs universitaires peuvent assumer des tâches d'enseignement, un seul groupe détient le titre de professeurs. Formentils pour autant un groupe spécifique ayant une identité professionnelle ? Conçoiventils leur occupation d'enseigner comme une carrière ? Ont-ils conscience d'appartenir à un groupe professionnel ? Cela n'est pas évident, du moins dans l'espace français, avant le $\mathrm{XV}^{\mathrm{e}}$ siècle. Les obstacles sont nombreux: institutionnel, social, économique. Poser ainsi le problème, c'est abandonner la conception substantialiste des groupes sociaux confinés dans une définition préalable et c'est mettre en avant, dans la suite des travaux de L. Boltanski', les mécanismes de formation des groupes professionnels, lesquels procèdent d'une conjoncture historique. Historiens et sociologues ne s'entendent pas toujours sur la définition à donner au concept de professionnalisation. S'attacher de trop près, par ailleurs, aux études sociologiques sur les groupes professionnels post-industriels risque d'entraîner des anachronismes. J'entends utiliser le terme profession pour désigner simplement une occupation réglementée et rémunérée s'exerçant sur une longue période de temps et en

\footnotetext{
${ }^{1}$ L. Boltanski, «Une question de sociologie. Introduction 2 », Les cadres. La formation d'un groupe social, Paris, Éd. de Minuit, 1982.
}

Cahiers de Recherches Médiévales, 18, 2009 
exclusivité, à laquelle on reconnaît une expertise et qui revêt un prestige et une autorité.

Je me propose ici d'examiner le processus de professionnalisation des professeurs à Caen aux $\mathrm{XV}^{\mathrm{e}}$ et $\mathrm{XVI}^{\mathrm{e}}$ siècles. Ce processus est étroitement lié au financement des institutions, différent pour chacune, et partant, à ses rapports avec les pouvoirs publics. Comme l'ont montré T. Kouamé et L. Tournier, qui ont étudié le phénomène à Paris à la fin du Moyen Âge, ce ne sont pas les professeurs euxmêmes qui s'organisent et se dotent de moyens pour constituer un groupe professionnel, mais bien les pouvoirs publics qui réglementent leurs pratiques et surtout assurent leur sécurité matérielle ${ }^{2}$. Ainsi, la réforme du cardinal d'Estouteville en 1452 constitue-t-elle un premier jalon vers la professionnalisation des régents parisiens. Prenant le relais de cette étude, je souhaite montrer qu'à l'université de Caen, les deux réformes que l'institution a connues au XVI ${ }^{\mathrm{e}}$ siècle représentent deux moments forts de la professionnalisation des régents de cette université de province. À partir du moment où l'enseignement est perçu comme un travail qui, comme tous les autres, mérite une rémunération et non plus dans sa gratuité, selon le principe que la science est un don de Dieu (et ne peut par conséquent être vendue), les efforts de professionnalisation émergent. La rémunération des professeurs, comme nous le verrons, n'est toutefois pas sans conséquence sur la dynamique entre les régents.

\section{Les professeurs de Caen, 1450-1550}

L'université de Caen est une institution de taille moyenne qui accueille annuellement entre 300 et 700 étudiants, du milieu du $X^{e}$ au milieu du $X^{\mathrm{e}} \mathrm{I}^{\mathrm{e}}$ siècle, et ses effectifs sont presque exclusivement d'origine normande $(90 \%)$. La riche documentation de Caen autorise une analyse sociologique des professeurs de 1450 à 1550. Pour cette analyse, j'ai tenté de mesurer la stabilité du corps professoral au sein de l'institution. La démonstration qui suit ne tient compte que des professeurs régents, c'est-à-dire que des individus qui détiennent le titre de docteur et qui ont les obligations des lectures ordinaires, de l'organisation des disputes, de la direction des étudiants et de l'attribution des grades. Il s'agit donc d'un groupe restreint d'universitaires qui ont dû remplir toutes les conditions d'accès à la régence et qui se distinguent des autres universitaires, bacheliers et licenciés, qui pouvaient également occuper des tâches d'enseignement ${ }^{3}$. À Caen, les professeurs régents avaient pour obligation de prêter serment chaque année devant l'assemblée générale, ce qui nous permet de les suivre ${ }^{4}$.

\footnotetext{
${ }^{2}$ T. Kouamé, L. Tournier, «L'honneur des docteurs. Statut social et identité professionnelle chez les universitaires parisiens à la fin du Moyen Âge ", Cahiers d'histoire, nº 71, 1998, p. 28-31.

${ }^{3}$ Dans le cadre restreint de cet article, il ne m'apparaît pas utile de m'étendre sur les diverses conditions d'accès au doctorat et à la régence. Voir J. Verger, "Teachers », A History of the University in Europe, t. I, H. de Ridder-Symoens (ed.), Cambridge, Cambridge Univ. Press, 1992, p. 144-147.

${ }^{4}$ Archives départementales du Calvados (désormais abrégé ADC), D 65 et D 66. Il arrive fréquemment que les procès-verbaux ne transcrivent pas le nom des régents, mais indiquent
} 
Nombre de professeurs à Caen (1457-1550)

\begin{tabular}{|l|c|c|r|}
\hline \multicolumn{1}{|c|}{ Facultés } & $\mathrm{XV}^{\mathrm{e}}$ siècle & $\mathrm{XVI}^{\mathrm{e}}$ siècle & Total \\
\hline Arts & 158 & 79 & 237 \\
\hline Médecine & 11 & 18 & 29 \\
\hline Droits & 31 & 11 & 42 \\
\hline Théologie & 35 & 19 & 54 \\
\hline Total & 235 & 127 & 362 \\
\hline
\end{tabular}

Malgré les lacunes de la documentation de Caen et le manque d'assiduité de certains professeurs à prêter serment, ces listes permettent d'apprécier l'importance des effectifs professoraux et de calculer la durée de leur carrière. Les professeurs de la faculté des arts sont les plus nombreux et les plus instables. Leur nombre varie en fonction du nombre des nouveaux maîtres ès arts et non en fonction du nombre d'étudiants inscrits aux matricules. En fait, jusqu'au milieu du XVI ${ }^{\mathrm{e}}$ siècle, ils sont en moyenne une quarantaine par année. Par la suite, malgré la hausse des immatriculations des étudiants, les effectifs professoraux à la faculté des arts chutent de moitié pour se stabiliser à une moyenne annuelle de 23 individus. Le trop grand nombre de régents à la faculté des arts signifie inévitablement un plus grand partage des droits perçus auprès des étudiants, ce qui explique sans doute l'abandon de la pratique de la régence obligatoire et la volonté, au $\mathrm{XVI}^{\mathrm{e}}$ siècle, de maintenir un nombre plus bas de régents. La régence obligatoire constitue un obstacle institutionnel à la professionnalisation dans la mesure où, en faisant de l'enseignement le prolongement obligatoire des études, elle a pour effet d'empêcher la délimitation d'un espace professionnel spécifique. Le nombre des professeurs des facultés supérieures est moins important et plus stable: 15 professeurs des facultés supérieures en moyenne prêtent serment annuellement. Les professeurs de la faculté de médecine sont les moins nombreux : entre 1487 et 1520 , leur nombre varie entre 1 et 3 . Il augmente à 5 en 1521 . La faculté de théologie compte une moyenne annuelle de 7 professeurs. En 1479, ils sont toutefois 12 à prêter serment. Le nombre de professeurs de la faculté de droits connaît une grande variation : il oscille entre 1 et 10 .

La documentation permet de suivre la trajectoire de 258 professeurs entre 1440 et 1550 . Dès le $X^{\mathrm{e}}$ siècle, un important noyau de professeurs, tant à la faculté des arts que dans les facultés supérieures, font des carrières durables à l'université. Le renouvellement des professeurs à la faculté des arts est constant, mais il demeure qu'une partie appréciable des effectifs du professorat est stable. On retrouve donc à Caen la même typologie que celle observée par J. Verger au Moyen Âge, à savoir, d'une part, un noyau durable de professeurs qui enseignent longtemps dans la même institution et, d'autre part, un autre groupe plus volatile ${ }^{5}$.

qu'ils ont prêté serment. Pour une analyse détaillée, voir L. Roy, L'université de Caen aux $X V^{e}$ et $X V I^{e}$ siècles. Identité et représentation, Leyde, Brill, 2006, p. 146 et sq.

5 J. Verger, "Les professeurs des universités françaises à la fin du Moyen Âge », Les universités françaises au Moyen Âge, Leyde, Brill, 1995, p. 181. 
Durée des carrières de professeurs $\left(\mathrm{XV}^{\mathrm{e}}\right.$ siècle-1 ${ }^{\text {ère }}$ moitié du $\mathrm{XVI}^{\mathrm{e}}$ siècle)

\begin{tabular}{|l|c|c|c|c|}
\hline \multicolumn{1}{|c|}{ Facultés } & $\begin{array}{c}\mathrm{XV}^{\mathrm{e}} \text { siècle } \\
1 \text { à } 10 \text { ans }\end{array}$ & $\begin{array}{c}\mathrm{XV}^{\mathrm{e}} \text { siècle } \\
11 \text { à }+20 \text { ans }\end{array}$ & $\begin{array}{c}\mathrm{XVI}^{\mathrm{e}} \text { siècle } \\
1 \text { à } 10 \text { ans }\end{array}$ & $\begin{array}{c}\mathrm{XVI} \\
11 \text { à }+20 \text { siècle }\end{array}$ \\
\hline Arts & $61 \%$ & $39 \%$ & $51 \%$ & $49 \%$ \\
\hline Médecine & $27 \%$ & $73 \%$ & $32 \%$ & $68 \%$ \\
\hline Droits & $58 \%$ & $42 \%$ & $16 \%$ & $84 \%$ \\
\hline Théologie & $63 \%$ & $37 \%$ & $65 \%$ & $35 \%$ \\
\hline
\end{tabular}

Ces pourcentages ne tiennent pas compte du fait que de nombreux professeurs des facultés supérieures ont commencé à enseigner à la faculté des arts, laquelle sert de tremplin, en quelque sorte, pour poursuivre des études dans les facultés supérieures, avant d'accéder au titre de docteur. Parmi les professeurs de la faculté des arts, plus de $20 \%$ ont ainsi poursuivi leur enseignement dans une autre faculté. Il s'agit surtout des théologiens : du $\mathrm{XV}^{\mathrm{e}}$ au milieu du $\mathrm{XVI}^{\mathrm{e}}$ siècle, $40 \%$ d'entre eux sont d'abord régents à la faculté des arts. C'est le cas pour $17 \%$ des juristes au $\mathrm{XV}^{\mathrm{e}}$ siècle et $9 \%$ au $\mathrm{XVI}^{\mathrm{e}}$ siècle. Dans le cas des professeurs de médecine, ils sont $9 \%$ à enseigner d'abord en art au XV siècle et plus de $29 \%$ à le faire au $\mathrm{XVI}^{\mathrm{e}}$ siècle.

Les premiers professeurs de Caen sont nommés directement par l'autorité royale. Henri VI, qui a fondé l'Université en 1432, attire à Caen surtout des universitaires d'origine normande pour enseigner dans la nouvelle université : cinq maîtres de l'université de Paris et deux professeurs célèbres, Thomas Basin et Pierre de la Hazardière, lesquels ne restent cependant que peu de temps à l'université. Un seul Anglais, Michel Tregorre, qui a fait ses études au Exeter College à Oxford, n'enseigne à Caen que quelques années. En 1436, un salaire est attribué aux professeurs, mais on ne sait pas s'il a été régulièrement versé durant le règne d'Henri $\mathrm{VI}^{6}$. Après cette période d'établissement, la nomination des professeurs de Caen, comme à Paris, appartient aux facultés. Les facultés supérieures contrôlent strictement le nombre de leurs régents, le plus souvent à la baisse, de façon à assurer aux docteurs en place des revenus plus substantiels et à ne pas avoir à partager leur autorité $^{7}$. Les facultés supérieures n'acceptent de créer de nouveaux docteurs que dans la mesure où il existe un poste vacant de professeur. Dès qu'ils prennent le titre de docteur, on les voit prêter serment comme régent. À mesure que l'Université s'organise, elle peut devenir plus autonome dans la reproduction de son corps

${ }^{6}$ En 1436, $\mathrm{M}^{\mathrm{e}}$ Pierre Gouier, professeur de droit canon reçoit 200 livres par an; $\mathrm{M}^{\mathrm{e}}$ Pierre Gerland et $\mathrm{M}^{\mathrm{e}}$ Jean Blondel, chargés de l'enseignement dans la faculté des arts, reçoivent chacun 100 livres. Ces sommes devaient être prises sur la saisie des rentes des collèges parisiens. Après la perte de ces revenus, Henri VI accorde, en 1448, 450 livres tournois pour le salaire de six professeurs. $\mathrm{M}^{\mathrm{e}}$ Jean Fleurie, docteur en théologie, $\mathrm{M}^{\mathrm{e}}$ Roger d'Estampes, docteur dans les deux droits, Richard du Moustier, chargé de l'enseignement en droit canon, $\mathrm{M}^{\mathrm{e}}$ Eudes Le Noble, licencié en médecine, reçoivent un traitement annuel de 80 livres, tandis que $\mathrm{M}^{\mathrm{e}}$ Guillaume de La Villette, docteur en droit civil, a un salaire de 100 livres et Jean du Val reçoit 30 livres pour l'enseignement des arts (ADC, 1 D 1135).

${ }^{7}$ J. Verger, «Teachers », art. cit., p. 147. 
professoral et ne confie bientôt les tâches d'enseignement qu'à des Normands. L'origine géographique est connue pour 165 professeurs : 161 étaient Normands, 4 seulement sont étrangers à la Normandie ${ }^{8}$. Une grande proportion de ces professeurs ont fait leurs études ou ont acquis leurs grades à Caen, soit 144 sur 239 professeurs $(60 \%)$. D'autres professeurs de Caen ont fait un séjour plus ou moins prolongé dans une autre université, notamment à Paris, avant de revenir y enseigner.

Tant que les professeurs ne reçoivent pas de salaire des pouvoirs publics, la faculté a pleine autorité sur le recrutement professoral, mais l'absence de salariat constitue un obstacle économique majeur à la professionnalisation du groupe des régents. La rémunération des professeurs est alors assurée par la perception des droits d'examen, par les collectes auprès des étudiants et par la participation à des activités de la corporation, comme les messes et les cérémonies de collation des grades. Quelques professeurs jouissent d'une prébende. Sur 239 professeurs, 90 (37\%) sont titulaires d'un bénéfice ecclésiastique. Les médecins vivent de leur pratique et les juristes font des consultations pour lesquelles ils reçoivent une rétribution. Les régents des facultés des arts tiennent une pension, enseignent dans les collèges, reçoivent de leurs étudiants les revenus des collectes et peuvent tirer des frais d'examens des montants substantiels. Ce système de rétribution multiple peut fonctionner tant que les étudiants sont assez nombreux, ce qui est apparemment le cas jusqu'à l'aube des guerres de Religion'. Ce système fonctionne aussi tant que l'on n'est pas trop exigeant à l'égard des professeurs.

\section{La réforme de 1521}

L’université de Caen connaît une première réforme de son institution par les autorités publiques au début du $\mathrm{XVI}^{\mathrm{e}}$ siècle. Ses objectifs généraux sont les mêmes que ceux des autres réformes imposées au $\mathrm{XV}^{\mathrm{e}}$ siècle $^{10}$ en insistant sur la discipline des étudiants et l'assiduité des professeurs. Ces réformes, selon J. Verger, visent essentiellement l'intégration plus étroite des universités à «l'ordre politique et sociale du royaume» en réduisant leurs privilèges et leur autonomie. Comme pour les autres réformes universitaires du $\mathrm{XVI}^{\mathrm{e}}$ siècle, celle de 1521 accompagne une restructuration de l'administration de la justice ${ }^{11}$. En effet, l'Échiquier de Normandie devient en 1515 un parlement souverain lequel s'investit rapidement de la mission de veiller à la qualité de l'enseignement du droit dans la province. Le parlement de Rouen nomme trois commissaires qui se rendent à Caen en juillet 1521, et un arrêt

\footnotetext{
${ }^{8}$ Outre Michel Tregorre, on retrouve Jean Rinelle, originaire de Paris ; Pierre de Castiglione originaire de Milan; Beroalde Marège de Brémont, originaire du diocèse de Clermont.

${ }^{9}$ Les collectes des professeurs de droit s'élèvent à 25 sous par étudiant, sans compter les différents frais d'examen, et les professeurs peuvent, par tous les moyens de droit, les forcer à les payer (ADC, $1 \mathrm{Mi} 49$, fol. $160^{\mathrm{r}}$ ).

${ }^{10} \mathrm{~J}$. Verger, "Les universités françaises au $\mathrm{XV}^{\mathrm{e}}$ siècle : crise et tentatives de réforme", Cahiers d'histoire, t. 21, 1976, p. 43-66.

${ }^{11}$ D. Julia et J. Revel, Histoire sociale des populations étudiantes, t. 2, Paris, EHESS, 1989, p. 108 .
} 
est rendu le mois suivant ${ }^{12}$. Le préambule de l'arrêt motive ainsi l'intervention du Parlement dans les affaires de l'Université, dans laquelle «y avoit plusieurs faultes et abbus, tant parce que les lectures n'y estoient ordinairement faictes par les docteurs et régens en chascune facultés de ladite Université que en plus exactions, et que ès collèges et maisons n'estoit tenu, ne exercé bonne discipline». Les maux et abus dénoncés par l'arrêt sont autant d'indicateurs de la non-professionnalisation des professeurs : leur manque d'assiduité, leur absentéisme, le cumul des occupations sont les problèmes cernés par l'arrêt, lesquels sont provoqués en grande partie par l'absence d'un salaire leur permettant de vivre exclusivement de leur métier. L'arrêt de la réforme fait en outre appel à une forme d'éthique professionnelle en encourageant le professeur à «faire bien son devoir».

$\mathrm{Au}$ moment de la réforme, l'enseignement du droit est donné par des licenciés. Afin de ne pas diminuer les revenus perçus auprès des étudiants, les professeurs des facultés de droits n'ont plus reçu de nouveaux docteurs et ont même refusé de pourvoir aux postes vacants. Il n'y a alors au collège des droits que trois professeurs, au demeurant fort pris par d'autres occupations, ce qui ne leur laisse guère de temps pour enseigner ${ }^{13}$. L'arrêt de la réforme veut revenir aux usages anciens et fixe le nombre de docteurs à six, dont trois professeurs de droit civil, deux de droit canon et un «institutaire». Les commissaires de la réforme exigent que soient faits docteurs des licenciés et ce, gratuitement, ce qui est une énorme concession. Parmi les candidats, François Cabart apparaît comme étant l'emblème du bon professeur: les commissaires admirent ses «sens, littérature, facunde et bonne discipline» et il s'est aussi montré «sçavant et éloquent». L'absentéisme et le manque d'assiduité des professeurs encouragent les commissaires à réglementer l'occupation. Les professeurs doivent dorénavant enseigner deux leçons par jour. Ils n'ont plus le droit de s'absenter sans motivation et doivent faire approuver leur substitut par le conservateur des privilèges royaux. On veut ainsi rendre l'occupation exclusive et on reconnaît aux professeurs une compétence spécifique. Ensuite, le temps académique devient plus structurée : les horaires et le calendrier ne sont pas laissés au hasard. Les commissaires inscrivent consciencieusement l'ordre et le contenu des leçons, inquiets de la qualité de l'enseignement du droit. En outre, ils ordonnent que soit installée une horloge sur le bâtiment de l'Université pour mettre fin aux retards des professeurs et des étudiants. Enfin, les commissaires s'attaquent au coeur du problème: la rémunération des professeurs. Les commissaires ordonnent à la ville de verser un salaire de 50 livres à chacun des quatre professeurs de droit et ils permettent aux régents de continuer à recevoir «l'escolage» de leurs étudiants.

L'arrêt de réforme ne résout pas tous les problèmes, loin s'en faut. La ville ne versera pas les 200 livres demandées par l'arrêt et ses dispositions ne sont pas toujours respectées. En 1530, une «grande multitude d'écoliers» se plaint au lieutenant du bailli de Caen, conservateur des privilèges de l'Université, d'un

12 A. Bénet, Inventaire des archives de l'université de Caen conservées aux Archives départementales du Calvados, t. I, Caen, H. Deslesques, 1892-94, p. 69-78.

${ }^{13}$ Pierre de Lesnauderie occupe l'office de scribe de l'Université ; Jean Gaudin est official de l'évêque de Bayeux et par conséquent conservateur des privilèges apostoliques et Marc Le Comte a une charge d'avocat. 
professeur de droit, René Patry, qui, malgré l'arrêt de la réforme, «ne faisait ni avait fait son devoir de lire deux fois par jour, ni même une fois par jour le plus souvent, et encore infructueusement, ledit Patry, présent, disant faire bien son devoir et non les autres docteurs du collège $\gg{ }^{14}$. Le lieutenant du bailli, Jean Malherbe, se transporte donc au collège des droits pour y entendre les griefs de la partie étudiante et du professeur, puis il réunit les universitaires au couvent des Cordeliers, lieu habituel des assemblées. L'accusé s'adresse à la cour du lieutenant du bailli «par paroles arrogantes », use de «paroles outrageantes» et de menaces envers les étudiants plaignants. Il accuse le scribe de l'Université d'être « son ennemi mortel». Il est dépeint comme un grossier personnage et c'est autant sa cuistrerie que son manque d'assiduité qui lui sont reprochés. Premier grief, le professeur Patry ne donne pas ses deux leçons par jour. Pour sa défense, il fait une «réponse arrogante ", à savoir qu'il «n'était pas sujet à faire deux lectures par jour, et qu'il était provectior ceteris doctoribus [...] voulant inférer qu'il était le plus savant, ainsi que expressément il disait devoir être entendu ledit terme provectior $»$. Il dit encore que les autres docteurs du collège ne font qu'une lecture et que «si on en veut avoir deux, qu'on cherche qui les fera ». Second grief, Patry arrive en retard à ses leçons. Il se fait remontrer par son collègue Cabart, « honnêtement et modestement » devant l'école. C'est alors qu'il l'insulte devant témoins en le traitant de "gros asne», faisant fi de l'arrêt qui recommande aux docteurs « de se contenir et de ne pas user de paroles injurieuses envers les autres docteurs ». Cabart demande réparation « vue cette grossièreté dudit Patry». Ainsi, contrairement à son collègue Cabart, Patry a tout du «mauvais professeur». Malgré les remontrances du lieutenant du bailli, Patry persiste dans ses mauvais comportements: il s'absente du collège sans permission et sans présenter de substitut à être approuvé. À l'issu de cette enquête, le lieutenant demande aux étudiants s'il y a d'autres professeurs qui méritent des remontrances. Ils affirment que non, « qu'ils étaient contents desdits autres docteurs, disant qu'ils faisaient bien leur devoir, excepté Patry $\rangle^{15}$.

La réforme de 1521 marque un premier jalon dans la volonté du Parlement de discipliner le corps professoral. Ce faisant, il induit inconsciemment une éthique professionnelle et permet l'émergence du processus de professionnalisation. Les paramètres s'affinent à la fin $\mathrm{du} \mathrm{XVI}^{\mathrm{e}}$ siècle, alors que 1'Université traverse une grave crise.

\section{La réforme de 1586}

Les guerres de Religion ont laissé l'Université dans un état de délabrement complet et très peu d'étudiants persistent à suivre leurs leçons. Des efforts importants de la ville et de la province ont dû être consentis pour la relever du marasme. Après l'enregistrement de l'ordonnance de Blois au parlement de Paris (janvier 1580), le parlement de Rouen s'investit dans une seconde réforme de l'Université, qui dure plus de six ans. Elle commence le 27 octobre 1580, alors que des commissaires dont Pierre Le Jumel, sieur de Lisores et président du parlement de Rouen, se rendent à Caen. Ils ouvrent d'abord une enquête et s'attachent à

\footnotetext{
${ }^{14}$ A. Bénet, op. cit., t. I, p. 78.

${ }^{15}$ Ibid., p. 80.
} 
connaître tant les exercices académiques et les moyens de subsistance des professeurs que l'état général des finances de l'Université.

L'interrogatoire du prieur du collège des droits est très instructif: Claude du Buisson fait savoir que le collège abrite cinq autres professeurs et que ce nombre n'est atteint que depuis trois ans. Il n'y a pas de professeur « institutaire», mais les licenciés se chargent de faire ces leçons. Il précise que les professeurs enseignent les deux droits et que la distinction entre droit civil et droit canon n'est plus conservée, « tant à raison de la nécessité du temps que pour le peu d'auditeurs qui se sont présentés aux lectures de droict canon ». Au sujet de l'ordre des lectures tel que défini par l'arrêt de la réforme de 1521, le prieur fait valoir qu'il est depuis longtemps délaissé, ce que déplorent les commissaires de la réforme qui reprochent aux professeurs d'agir à leur guise. En ce qui concerne la durée des études, les commissaires blâment le prieur de ne pas respecter les temps requis par l'arrêt. S'ils ne gardent pas deux ans les bacheliers et trois ans les licenciés, ils n'arriveront qu'à «graduer les ingnorans et incogneüz». Il y a en effet «ung grand murmure et scandalle par toulte ceste province » disant que des hommes, qui ne connaissent pas un seul mot de latin, sont promus licenciés et occupent des charges d'officiers du roi dans les cours souveraines et inférieures, «au plus grand préjudice du bien public». À quoi le prieur répond que s'il faut respecter les temps d'études, «il fauldroyt quicter leur profession, pour ce qu'il n'a poinct de gaiges, ny aultres esmolumentz, et qu'il n'avoit autre moien de vivre». Il ajoute toutefois que ceux qui ont été promus aux grades, ont été jugés dignes du degré. Sur la question des droits perçus auprès des étudiants, le professeur Jean Paon précise que l'inflation suit immanquablement l'augmentation du nombre de professeurs: lorsque que les régents n'étaient qu'au nombre de deux, ils se contentaient de demander aux licenciés 6,7 ou 8 écus, «mais depuis deux ans qu'ilz sont cinq, tant en droit civil que canon, sont contrainctz de prendre jusques à 10 écus ». Par suite de l'enquête, le président Lisores reconnaît que ce qui manque à l'Université est le financement pour « gager des lecteurs et professeurs, affin d'y atirer escholiers et par ce moien rendre cette université plus fameuse $»^{16}$. Mais les bourgeois craignent de nouveaux impôts. On décide alors de demander au roi un octroi sur le sel et le revenu d'une prébende sur chaque église. Ce qui leur est accordé par Henri III en $1581^{17}$. Ces revenus doivent servir à payer le salaire des professeurs de cinq facultés et des collèges qui enseignent les lettres grecques et hébraïques, ainsi que les mathématiques.

L'arrêt de réforme est émis tardivement, en octobre $1586^{18}$. Il compte 41 articles, dont la moitié s'attardent à encadrer et à définir les tâches des professeurs, principaux agents de la renommée de l'Université. Plus détaillé que l'arrêt de 1521, celui de 1586 aborde des problèmes similaires, mais sur un autre ton : plus d'ordre, plus de discipline, plus de surveillance sur chacun des membres du corps académique, à l'image de la pédagogie des collèges jésuites. L'assiduité des professeurs et l'absentéisme demandent toujours à être surveillés : les principaux des

${ }^{16} \mathrm{H}$. Prentout, Renovatio ac reformatio in Universitate Cadomensi per XVI saeculum, Caen, 1901, p. 108.

${ }^{17}$ Les démarches pour faire respecter cet octroi ont été très longues. Voir L. Roy, op. cit., p. 214 et sq.

${ }^{18}$ A. Bénet, op. cit., t. I, p. 94 (D 51), plaquette petit in $-4^{0}$ de 38 pages sur papier. 
collèges ne peuvent s'absenter sans la permission du conservateur des privilèges royaux et doivent être remplacés par des personnes «suffisans et capables, tant de doctrine que bonne mœurs, et non mariez ». Les professeurs qui ne donnent pas leurs leçons et abandonnent leur classe au cours de l'année scolaire, sont susceptibles de payer une amende, d'être privés des privilèges de l'Université « et déclarez indignes de faire lecture en icelle ». Les professeurs de droit doivent toujours donner leurs deux leçons par jour, et leur présence est obligatoire lors de la collation des grades. L'arrêt ajoute des articles sur la décence des vêtements de tous les universitaires et sur l'obéissance due aux supérieurs hiérarchiques (principaux des collèges, recteur et conservateur des privilèges royaux). Comme en 1521, l'arrêt de 1586 précise l'horaire et le calendrier des leçons, ainsi que les obligations académiques des professeurs (présence aux examens, par exemple). Toutefois, la préoccupation de la pédagogie est beaucoup plus marquante et plus développée: la composition en langue française, la dictée, le théâtre, la civilité sont autant de méthodes pour assurer la formation des jeunes. Quant aux professeurs, ils doivent se montrer compétents dans leur enseignement: ils seront «faciles et clairs en leurs leçons, sans affecter l'obscurité et admonester souvent les jeunes disciples de leur devoir ». On répète dans plusieurs articles que les professeurs doivent être des hommes «doctes», "propre à l'instruction et utilité des estudians». Enfin, l'arrêt reconnaît la rémunération des professeurs comme une évidence : les régents reçoivent un salaire «selon leur labeur, diligence et mérite ». Ils ne peuvent exiger d'autres rétributions de leurs élèves, à part un demi écu à la Saint-Martin et un écu lors des fêtes de la Pentecôte «pour recongnaissance de la bonne diligence et vigilance desdist régents ». Tous les ans, au mois de septembre, le bailli et les échevins ont pour tâche d'examiner les professeurs, de remplacer ceux qui ne font pas l'affaire et de décider de leur gage, « selon leur mérite».

Le salaire des professeurs est assuré par l'octroi sur le sel accordé par le roi et diverses donations. Ces deniers sont gérés par le receveur de la ville, mais le rendement est fluctuant. Aussi, les professeurs ont-ils dû batailler pour toucher leur salaire qu'ils trouvent, au demeurant, bien modeste. Le principal du collège du Bois, Jean Colet, déclare en octobre 1585 à l'Hôtel de ville qu'il a de la difficulté à recruter des professeurs, à cause du «scandale qui a été de ce que, en l'année dernière, ils ont été mal payés » ${ }^{19}$. Lors de l'assemblée du conseil de ville du 23 septembre 1586, qui entérine l'arrêt de réforme, le même Colet demande une augmentation de ses gages, «sinon déclare préférer remettre sa charge ${ }^{20}$. Les échevins promettent vaguement d'augmenter les salaires à l'avenir. Les plaintes des professeurs s'abattent bientôt sur le conseil de ville ${ }^{21}$. Les frustrations des professeurs sont d'autant plus grandes que des gages nettement supérieurs aux leurs

\footnotetext{
${ }^{19}$ Ibid., t. II, p. 98 (D 197).

${ }^{20} \mathrm{Ibid}$.

${ }^{21}$ En 1586, Claude du Buisson demande au nom d'un de ses fils ses gages pour l'année entière (85 livres); Jacques de Cahaignes demande en 1596 une augmentation de salaire, laquelle lui a été accordée en "considération de services, suffisance et insigne doctrine du sieur de Cahaignes »; il obtient 100 livres de gages. En 1588, le fils de Jean Rouxel réclame les 500 livres de gage de son défunt père (G. Dupont, Registre de l'Hôtel de ville de Caen. Inventaire manuscrit, Caen, 1888, t. II, p. 135, 140, 163).
} 
sont attribués à des professeurs étrangers. L'arrivée de professeurs étrangers, mieux payés, provoque en effet d'importantes tensions à l'Université. Les professeurs locaux réprouvent de se faire déclasser par des nouveaux venus, perçus par les autorités publiques comme les garants de la relance de l'Université.

Dès 1582, les négociations commencent pour recruter des professeurs célèbres, capables d'attirer à Caen de nombreux étudiants. À l'instar des universités de Valence et de Bourges, dont la renommée repose sur l'enseignement des plus grands juristes du royaume, la ville de Caen veut, elle aussi, investir dans un professeur de droit, sans toutefois avoir les moyens de sa politique. Les échevins confient à François d'O, lieutenant général de la Basse Normandie, d'approcher Cujas, qui est alors à Bourges ${ }^{22}$. La ville lui offre 500 écus, mais Cujas refuse l'invitation ${ }^{23}$. L'entrevue d'embauche de Daniel Hotman est l'occasion de mettre à l'épreuve certains critères de compétence. En octobre 1583, les échevins de Caen sont avertis que Daniel Hotman, âgé de 25 ans, fils aîné du célèbre juriste François Hotman et converti au catholicisme, cherche une chaire d'enseignement ${ }^{24}$. Ils le font venir à Caen et lui font faire des leçons, «affin que on peust juger de sa suffisance et érudition ». Durant trois jours, Hotman fils s'acquitte de sa tâche devant les officiers royaux de justice et des finances, les autorités urbaines, les universitaires, les écoliers et les bourgeois de la ville. Le corps des élus fait ensuite un rapport au président du Parlement dans lequel il reconnaît son incompétence à juger des qualités intellectuelles du jeune homme. Il se tourne plutôt vers le jugement des universitaires et des officiers de justice, qui «plus s'y congnoissoient». Les avis étaient partagés et rendent compte des tensions provoquées par l'arrivée d'un professeur venu de l'extérieur. Hotman est alors :

\begin{abstract}
tenu pour jeune homme fort modeste et de bonne espérance. Néantmoins aucuns disent qu'il est encore peu exercité et a peu de hardiesse pour enseigner publiquement. Les autres excusent cesdits deffaults sur son jeune âge et que en continuamt, il se formera ou enhardira incontinent. Autres ont trouvé maulvais que en toutes ses trois leçons, il a tousjours continuellement leü, ce qu'il a prononcé qu'il avait par escript devant luy. Autres passent plus oultre dizants qu'ils ne tiennent en ses leçons plus d'erudition que en icelles des autres qui estoient desja avec nous $^{25}$
\end{abstract}

Le manque d'enthousiasme de certains n'empêche pas l'embauche de Daniel Hotman pour l'année, au gage de 50 écus. Les échevins ne semblent toutefois pas convaincu que le jeune professeur allait à lui seul restaurer la réputation de l'Université. Ils achèvent leur lettre au président du Parlement par ces mots : «s'il estoit trouvé d'appeler encores quelques autres professeurs en nostre université, il seroit très requis que ce fussent personnages bien congneüs et d'insigne érudition et consommé en la science en laquelle ils entendent faire profession, la renommée

\footnotetext{
${ }^{22}$ G. Dupont, op. cit., t. II, p. 15.

${ }^{23}$ Son salaire à Bourges est de 1600 livres par année. D. Hickey, «L'Université de Valence au $\mathrm{XVI}^{\mathrm{e}}$ siècle et son rayonnement intellectuel », Revue drômoise, t. 88, $\mathrm{n}^{\circ} 470,1993$, p. 569.

${ }^{24}$ G. Dupont, op. cit., t. II, p. 51.

${ }^{25}$ H. Prentout, Renovatio ac reformatio..., p. 111.
} 
desquels peüst attirer les auditeurs et exciter les particullers à bien faire à ladite université ». Le président leur répond que ses efforts pour trouver un professeur de droit sont limités par les moyens de la ville: «il ne s'en est trouvé qu'un ou deux en ce royaulme à la rémunération desquels vos moiens n'ont semblé suffisant ${ }^{26}$ ». Durant les deux années suivantes, la recherche de professeur de droit se poursuit ${ }^{27}$. L'embauche du professeur Claude Fournier cristallise les frustrations des professeurs qui ne sont plus payés. Celui-ci a enseigné à l'université de Dole en Franche-Comté et a obtenu d'assez bonnes conditions pour professer à Caen, soit un salaire de 500 livres par an. En 1595, Jacques de Cahaignes demande à la ville d'annuler son contrat «à cause de l'insolence et déportement du sieur qui troublent la paix de l'Université ». En 1597, un arrêt de la cour du Parlement lui attribue des gages de 333 écus par an et condamne la ville à lui payer 416 livres $^{28}$. Ces exigences sont préjudiciables aux autres professeurs qui ne reçoivent pas leur salaire depuis l'année précédente. Fournier quitte Caen pour Angers, mais maintient ses poursuites contre la ville ${ }^{29}$.

L'attribution d'un salaire aux professeurs et la recherche de la renommée changent la dynamique à l'Université: la reproduction du corps professoral ne fonctionne plus à partir des mêmes critères qu'auparavant. Les rapports entre l'Université et la ville se modifient également. Tout le XVII ${ }^{\mathrm{e}}$ siècle est ponctué de procès devant le Parlement entre la ville et l'Université concernant les gages des professeurs. En 1609, les échevins de la ville plaident devant la cour du Parlement que les professeurs de l'Université ont été corrompus par l'appât du gain et condamnent leur arrogance à prendre pour acquis ce «privilège » :

Avant 1580, l’Université était composée de plusieurs bons et capables docteurs et professeurs en toutes facultés, qui toutefois n'avaient aucuns gages, profits, ni émoluments, sinon ce qui pouvait provenir de la libéralité des écoliers et licences. L'octroi de $10 \mathrm{~d}$. par minot de sel fut un fonds de gages : bien qu'il n'ait été concédé que pour un temps, les défendeurs veulent contraindre la ville à leur continuer leurs gages annuellement, comme si c'était chose fondée et assurée. Depuis les derniers troubles, 3 ou 4 jeunes docteurs peu savants, par brigues et menées, se sont coulés au corps de ladite université, l'ont remplie de factions, y ont apporté l'ignorance et l'oisiveté, et néantmoins à outrance tirent et courent après les gages, de telle sorte

${ }^{26} \mathrm{Ibid}$, p. 112.

${ }^{27}$ François d'O propose, en juin 1584, à M. Viliard de devenir professeur de droit civil, mais personne ne connaît sa réputation. En juillet 1584, Pierre Le Jumel suggère François Roaldès, célèbre juriste de Valence, mais la ville n'a toujours pas les moyens de satisfaire à ses conditions. En septembre 1586, les échevins de la ville demandent au professeur caennais Marin du Vicquet «d'envoyer quelque docte personnage professeur de loix et de faire mettre des affiches à Paris pour trouver ceux qui voudrait accepter cette charge ». Pierre Le Jumel trouve enfin un candidat: un Écossais nommé Bruce considéré «fort docte ès lois et en grec et les écoliers s'en contentent beaucoup ». D'autres professeurs extérieurs à Caen sont engagés à la faculté de droit: en 1593, Robert Godefroy accepte la charge de professeur en droit et la ville organise son déménagement (G. Dupont, op. cit., t. II, p. 338).

${ }^{28}$ A. Bénet, op. cit., t. II, p. 86-87 (D 181).

${ }^{29}$ Ibid., t. II, p. 89-90 (D 184). 
que depuis n'ont cessé de faire des procès et plaider contre les échevins, ont chassé les gens doctes de toutes parts et rempli les places d'ignorance et de paresse ${ }^{30}$.

La délimitation de l'espace professionnel des professeurs doit être replacé dans le contexte politique de l'émergence de l'État moderne et dans le contexte religieux de la contre-réforme. Les nouveaux besoins administratifs de l'État exigent des serviteurs compétents, ce qui l'amène à surveiller plus étroitement l'enseignement du droit. De même, la rivalité des confessions religieuses pousse l'État à contrôler strictement l'enseignement dispensé dans les universités et le recrutement des professeurs pour s'assurer de leur orthodoxie. La professionnalisation des professeurs exige d'abord qu'ils soient nombreux à exercer leur métier sur une longue période de temps. Mais pour qu'ils envisagent l'enseignement comme une carrière, d'autres conditions sont essentielles : un salaire, l'exclusivité de l'activité et la reconnaissance de critères de compétence.

Le concept de professionnalisation appartient, bien entendu, à nos catégories d'analyse actuelles. Mais les acteurs percevaient les choses différemment : l'action autoritaire des pouvoirs publics visait à discipliner le corps professoral. Or, cette discipline oblige la définition de critères qui, parce qu'ils sont ou non respectés, interviennent dans l'évaluation de la compétence du professeur, déterminant son mérite et donc sa rétribution salariale. Du même coup, venant d'une autorité extérieure à l'université - par le biais des réformes de 1521 et 1586 , intervenant jusque dans l'organisation du travail, le contenu des cours, l'obéissance hiérarchique - et combinée à une rétribution de plus en plus réglementée et reconnue, cette "disciplinarisation » du corps professoral contribue en même temps à sa définition propre et s'est effectuée au détriment de l'autonomie relative des individus et de l'institution. Ainsi, dans un contexte où il devient nécessaire de préciser et d'encadrer la tâche de professeur, il n'est pas étonnant que la pédagogie devienne une préoccupation, parce qu'elle est précisément spécifique et exclusive au corps professoral, ce qui permet de le caractériser sur le plan symbolique et professionnel. Aussi, ne faut-il pas négliger le rôle de la rétribution symbolique qui participe à cette professionnalisation en donnant au groupe des professeurs sa cohésion et sa cohérence, rétribution aussi bien pour les personnes que pour l'institution elle-même. On sait déjà que la dignité doctorale participait au prestige des professeurs, qu'ils avaient de nombreux privilèges, que plusieurs professeurs se sont engagés à long terme dans une carrière d'enseignement, malgré une faible rémunération, que l'attitude d'un professeur pouvait être contestée, jouant ainsi sur sa réputation, etc. Certes, la recherche d'une renommée par les autorités urbaines a imposé de délier les cordons de la bourse, mais cela n'interdit pas de se demander comment le prestige et sa simple quête par les individus et l'institution ont pu compenser, du moins en partie, l'insuffisance des émoluments et des fonds.

Lyse Roy

Université du Québec à Montréal Centre interuniversitaire de recherche en science et technologie

${ }^{30} \mathrm{Ibid}$. 